\title{
Single-photon single-ion interaction in free space configura- tion in front of a parabolic mirror
}

\author{
Magdalena Stobińska \\ Institute of Theoretical Physics and Astrophysics, University of Gdansk, ul. Wita Stwosza \\ 57, 80-952 Gdansk, Poland \\ Institute of Physics, Polish Academy of Sciences, Al. Lotników 32/46, 02-668 Warsaw, \\ Poland \\ E magdalena.stobinska@gmail.com \\ Robert Alicki \\ Institute of Theoretical Physics and Astrophysics, University of Gdańsk, ul. Wita Stwosza \\ 57, 80-952 Gdańsk, Poland
}

(Received

\begin{abstract}
The efficient interaction between single photons and single matter objects in free space is of key importance for quantum technologies. An experimental setup for testing this possibility involves single two-level ion trapped at the focus of a parabolic metallic mirror. We study the conditions for the setup, under which the assumption about the freespace mode structure of the radiation field in the vicinity of the atom is justified. In our analysis we apply vectorial properties of light by including polarization degree of freedom. We look for possible changes in the spontaneous emission rate of the atom resulting from the presence of the parabolic boundary conditions.
\end{abstract}

\section{Introduction}

The efficient interaction between single photons and single matter objects is of key importance for quantum technologies. Although, quantum electrodynamics is one of the best physical theories, this interaction remains an intriguing research from the fundamental point of view.

The strong matter-light coupling has applications in many branches of physics and technology: in quantum communication for quantum repeaters (coupling between the flying and stationary qubit), quantum computing, distributed networks [1, 2, 3] and microscopy [4, 5, 6]. It is achieved in cavity QED, where light is reflected from its mirrors and interacts with an object inside the cavity for a long time. The cavity supports only one (or very few) radiation mode and in this way prevents from unwanted interaction with other modes, which makes the evolution of the object difficult to invert. How- 
ever, technological solutions based on cavities QED are not scalable. Strong atom-light interaction in free space configuration [7, 8, 9, 10] i.e. without a cavity, may provide us with less technologically demanding solutions for large distances.

Possibility of such near perfect coupling in free space has already been theoretically predicted [10]. It can be achieved if the photon is prepared in a wavepacket with a suitable spatio-temporal profile. An experimental setup for testing this theory is currently being built. It involves single twolevel ion trapped at the focus of a parabolic metallic mirror (see Fig. 1) [11. The mirror with an additional electrode constitutes a Paul trap. Its parabolic shape allows to use it as an electric field mode converter, useful for shaping the photon wavepackets. However, numerous discussions arose in scientific community whether the mirror, being a half-cavity, indeed ensures a free space configuration. Similar work considering effects of the large cavity limit [12] and structure of standing light waves in half-cavity arrangement on atom decay rate was theoretically investigated within the scalar light model [13, 14. The change of density of modes near the atom in front of a planar mirror was experimentally verified [15].

In this paper we study the conditions for the setup in Fig. 1, under which the assumption about the free-space mode structure of the electromagnetic field in the vicinity of the atom is justified. In our analysis we apply vectorial properties of light by including polarization degree of freedom. We look for possible changes in the spontaneous emission rate of the atom resulting from the presence of the parabolic boundary conditions.

We emphasis that the setup under discussion is especially useful for quantum communication applications, since it allows for error correction schemes: the shape of the mirror ensures the access to all environmental degrees of freedom. This distinguishing feature of the setup makes also possible investigation of the universal model of spontaneous emission process in free space from the fundamental point of view. It is irreversible for the atomic subsystem alone, but unitary for the system as a whole [16].

This paper is organized as follows. In section 2. we discuss the setup and develop the formalism of the normal modes genuine to the parabolic geometry, without the mirror. In section 3. we analyze the correction to the spontaneous emission rate resulting from presence of the parabolic metallic mirror. We finish the paper with the conclusions.

\section{Decay rate in parabolic geometry}

Let us start the discussion with the description of the parabolic mirror ion trap depicted in Fig. 1. The ion is located in half-open space, or in a 


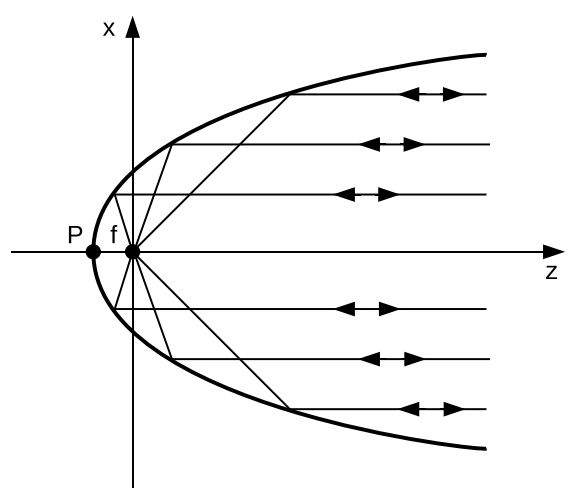

Fig. 1: The experimental setup under consideration: a single two-level ion is trapped at the focus of a parabolic metallic mirror. The focal length equals $f$. The ion interacts with the light coming from the whole $4 \pi$ solid angle.

half-cavity. The parabolic shape of the mirror ensures that if a light beam is sent parallel to the mirror axis towards the ion, it interacts with the light coming from the whole $4 \pi$ solid angle, and similarly it allows to collect the whole light resulting from its spontaneous decay. Thinking in terms of the classical ray-picture, the incident angle for either incoming or outcoming light is never equal to $\pi / 2$ (except from one direction). It means that if the atom emits a photon in the spontaneous emission process, contrary to the standard cavity case, the radiation is never back reflected to the atom, and thus the atom does not feel the presence of the boundary conditions, just like in free space. Nevertheless, the mirror creates the nodes and antinodes in the reflected modes and thus is able to change the electromagnetic vacuum structure. Therefore, in the limit where the focal length $f$ of the mirror is comparable to the wavelength $\lambda$ of the mode resonant with the atomic transition, similarly to a small cavity or in front of a planar mirror [17, the changes of the spontaneous decay rate should be significant. We will show that depending on the characteristic parameters of the setup: the focal length $f$, the wavelength $\lambda$ and the orientation of the atomic electric dipole moment, the setup provides us with either a free-space configuration or a tailored electromagnetic reservoir near the atom.

We begin with introducing our formalism of the normal modes genuine to the parabolic geometry without the mirror. Since the ion is located in a halfopen space we work within the framework of the Weisskopf-Wigner model of interaction between a single matter qubit and a quantized radiation field. We begin with the expansion of the electric field operator for the radiation field 
in free space using the basis (modes) suitable for the parabolic symmetry of the problem. We follow the results of [18, and use the modes given by the formula

$$
\vec{E}_{k, \ell, \mu}^{\sigma}(\vec{r})=\frac{k}{(2 \pi)^{3 / 2}} \int_{S^{2}} d \vec{n} e^{i k \vec{n} \cdot \vec{r}} h_{\ell, \mu}(\vec{n}) \vec{e}^{\sigma}(\vec{n}),
$$

where $\vec{k}=k \vec{n}$ denotes the wavevector, $\sigma=1,2$ enumerates polarization states, parameters $\ell=0, \pm 1, \pm 2, \ldots$ and $\mu \in(-\infty,+\infty)$ are the mode numbers. The unit vector $\vec{n}$ and the polarization vectors $\vec{e}^{1}(\vec{n}), \vec{e}^{2}(\vec{n})$ constitute the orthonormal basis what ensures the transversality condition

$$
\nabla \cdot \vec{E}=0
$$

We choose

$$
\begin{aligned}
\vec{n} & =(\sin \theta \cos \varphi, \sin \theta \sin \varphi, \cos \theta), \\
\vec{e}^{1}(\vec{n}) & =(\sin \varphi,-\cos \varphi, 0), \\
\vec{e}^{2}(\vec{n}) & =(\cos \theta \cos \varphi, \cos \theta \sin \varphi,-\sin \theta) .
\end{aligned}
$$

The manifest form of the modes $h_{\ell, \mu}(\vec{n})$ reads [18]

$$
h_{\ell, \mu}(\theta, \varphi)=\chi_{\mu}(\theta) \frac{e^{i \ell \varphi}}{\sqrt{2 \pi}}
$$

where

$$
\chi_{\mu}(\theta)=\frac{\exp (-i \mu \ln [\tan \theta / 2])}{\sqrt{2 \pi} \sin \theta}
$$

One can easily check the orthogonality and completeness conditions

$$
\begin{aligned}
& \int_{0}^{2 \pi} d \varphi \int_{0}^{\pi} d \theta \sin \theta h_{\ell, \mu}^{*}(\theta, \varphi) h_{\ell^{\prime}, \mu^{\prime}}(\theta, \varphi)=\delta_{\ell \ell^{\prime}} \delta\left(\mu-\mu^{\prime}\right), \\
& \sum_{\ell=-\infty}^{+\infty} \int_{-\infty}^{+\infty} d \mu h_{\ell, \mu}^{*}(\theta, \varphi) h_{\ell, \mu}\left(\theta^{\prime}, \varphi^{\prime}\right)=\delta\left(\varphi-\varphi^{\prime}\right) \frac{\delta\left(\theta-\theta^{\prime}\right)}{\sin \theta} .
\end{aligned}
$$

Combining Eq. (1) with Eq. (8) one obtains the orthogonality of the electric modes

$$
\int d^{3} \vec{r} \vec{E}_{k, \ell, \mu}^{* \sigma}(\vec{r}) \cdot \vec{E}_{k^{\prime}, \ell^{\prime}, \mu^{\prime}}^{\sigma^{\prime}}(\vec{r})=\delta\left(k-k^{\prime}\right) \delta\left(\mu-\mu^{\prime}\right) \delta_{\ell \ell^{\prime}} \delta_{\sigma \sigma^{\prime}}
$$

Let us consider an atomic qubit at a fixed position $\vec{r}$ in free space with the transition dipole parallel to the $z$-axis. Its excited and ground state are 
denoted by $|e\rangle$ and $|g\rangle$ respectively. The atom interacts with the quantized radiation field distributed over a continuum of modes centered around the optical atomic transition frequency $\omega_{0}$ and given by Eq. (11). The standard dipole-interaction Hamiltonian of such matter-field system reads $H=-\vec{d}$. $\hat{\vec{E}}(\vec{r})$ and simplifies for $\vec{d}=d \vec{e}_{z}$ to the following form

$$
H_{\text {int }}=-i d \sqrt{\frac{\hbar c}{2 \epsilon_{0}}} \sum_{\sigma, \ell} \int_{0}^{\infty} d k \sqrt{k} \int_{-\infty}^{+\infty} d \mu\left\{\vec{E}_{k, \ell, \mu}^{\sigma}(\vec{r}) \vec{e}_{z} \hat{\sigma}^{+} \hat{a}_{k, \ell, \mu}^{\sigma}-\text { h.c. }\right\},
$$

where $\hat{\sigma}^{+}=|e\rangle\langle g|$ and $\hat{\sigma}^{-}=|g\rangle\langle e|$ are the atomic rising and lowering operators respectively. This Hamiltonian shows that the radiation field couples to the atom only if its polarisation has a component which is parallel to the $z$-axis at the position of the atom so that $\vec{e}^{\sigma}(\bar{n}) \cdot \vec{e}_{z} \neq 0$. From now on, we fix the frequency of the mode, $k=\omega_{0} / c$. The spontaneous emission decay rate for the atom immersed in the electromagnetic field reservoir computed in the standard lowest order (Born) approximation equals

$$
\begin{aligned}
\Gamma(k, \vec{r})= & \frac{1}{(2 \pi)^{2}} \frac{d^{2} k^{3}}{2 \hbar \epsilon_{0}} \sum_{\sigma, \ell} \int_{-\infty}^{+\infty} d \mu \int d \vec{n} \int d \vec{n}^{\prime} f_{k}(\vec{n}, \vec{r}) f_{k}^{*}\left(\vec{n}^{\prime}, \vec{r}\right) \\
& \cdot e_{z}^{\sigma}(\vec{n}) e_{z}^{\sigma}\left(\vec{n}^{\prime}\right) h_{\ell, \mu}^{*}(\vec{n}, k) h_{\ell, \mu}\left(\vec{n}^{\prime}, k\right) .
\end{aligned}
$$

We denote here by $f_{k}(\vec{n}, \vec{r})$ the plane wave $e^{i k \vec{n} \cdot \vec{r}}$ and $e_{z}^{\sigma}(\vec{n})=\vec{e}^{\sigma}(\vec{n}) \cdot \vec{e}_{z}$ and use the fact the the summation over $\ell$ produces $\delta\left(\varphi-\varphi^{\prime}\right)$ (see Eq. (9) ). Therefore relevant $\vec{n}, \vec{n}^{\prime}$ and the $z$-axis belong to the same plane what leads to the formula

$$
\sum_{\sigma} e_{z}^{\sigma}(\vec{n}) e_{z}^{\sigma}\left(\vec{n}^{\prime}\right)=e_{z}^{2}(\vec{n}) e_{z}^{2}\left(\vec{n}^{\prime}\right)=\sin \theta \sin \theta^{\prime}
$$

Taking into account that the integration over $\mu$ yields another Dirac delta $\delta\left(\theta-\theta^{\prime}\right)$ we obtain

$$
\Gamma(k ; x, y, z)=\frac{1}{(2 \pi)^{2}} \frac{d^{2} k^{3}}{2 \hbar \epsilon_{0}} \int_{0}^{2 \pi} d \varphi \int_{0}^{\pi} d \theta \sin ^{3} \theta\left|f_{k}(x, y, z ; \varphi, \theta)\right|^{2},
$$

where $\vec{r} \equiv(x, y, z)$ and $f_{k}(\vec{n}, \vec{r}) \equiv f_{k}(x, y, z ; \varphi, \theta)$. Knowing that $\left|f_{k}(x, y, z ; \varphi, \theta)\right|=$ 1 , one recovers the standard result

$$
\Gamma_{0}(k)=\frac{1}{3 \pi} \frac{d^{2} k^{3}}{\hbar \epsilon_{0}}
$$




\section{Decay rate at the presence of a mirror}

The presence of the conducting parabolic mirror leads to the boundary conditions which should be imposed on the modes of the electric field given by Eq. (11). Since it is very challenging to solve the Helmholtz equation while keeping the zero-divergence condition (Eq. (2) ) and the boundary conditions satisfied at the same time [19], contrary to reference [19] we decided to keep the transversality condition at the cost of relaxing the precise value of the mode function on the mirror surface. The transversality condition relates the electric field to the geometry of the system and therefore, contributes to some geometrical factor present in the decay rate formula. The boundary condition ensures the discreteness of the normal modes. However, the system is so large that the density of modes can be approximated by a continuous spectrum. Enlarging the system, thus changing the boundary conditions slightly, will not influence the decay rate. Moreover, the boundary conditions are known exactly only for stationary fields.

We first introduce the parabolic coordinates $(\xi, \eta, \varphi)$ related to Cartesian ones in the following way

$$
\begin{aligned}
& x=2 \sqrt{\xi \eta} \cos \varphi \\
& y=2 \sqrt{\xi \eta} \sin \varphi \\
& z=\xi-\eta .
\end{aligned}
$$

The shape of the parabolic mirror is given by the equation

$$
\eta=f
$$

The normal modes (in fact their scalar counterparts) obtained by separation method and expressed in parabolic coordinates are given by products of the functions of $\xi, \eta, \varphi$, respectively [18]. We are interested in the $\eta$-dependent part $F_{\ell, \mu}(k ; \eta)$ which possesses the following asymptotic behaviour

$$
F_{\ell, \mu}(k ; \eta) \sim \frac{\cos \left\{\mu \ln 2 k \eta+k \eta-\alpha_{\ell, \mu}\right\}}{\sqrt{\eta}},
$$

where $\alpha_{\ell, \mu}$ is a certain phase. The boundary condition imposed on Eq. (20) at the value $\eta=f$ can be satisfied for a discrete set of $\mu_{m}$ only, which is related to the periodicity of the cosine function. We consider the simplest choice

$$
k f-\alpha_{\ell, \mu}=0, \mu_{m} \ln 2 k f=m \pi, m=1,2,3, \ldots
$$

This periodic condition is consistent with the replacement of the continuous 
set of modes given by Eq. (17) by a discrete one

$$
\begin{aligned}
\tilde{\chi}_{m}(\theta) & =\frac{\sin \left(\frac{m \pi \ln [\tan \theta / 2]}{\ln 2 k f}\right)}{\sqrt{2 \pi \ln 2 k f} \sin \theta} \text { for } \theta \in\left[\theta_{0}, \pi-\theta_{0}\right] \\
& =0 \text { otherwise }
\end{aligned}
$$

such that

$$
\tan \frac{\theta_{0}}{2}=\frac{1}{2 k f}=\frac{1}{4 \pi} \frac{\lambda}{f}
$$

The limitation for the $\theta$ angle results from the quantization condition and the fact that at the boundary the normal modes vanish $\tilde{\chi}_{m}\left(\theta_{0}\right)=0$. This anzatz modifies the formula for the decay rate because the completeness condition now reads

$$
\sum_{m} \tilde{\chi}_{m}(\theta) \tilde{\chi}_{m}\left(\theta^{\prime}\right)=I_{\left[\theta_{0}, \pi-\theta_{0}\right]}(\theta) \frac{\delta\left(\theta-\theta^{\prime}\right)}{\sin \theta}
$$

where $I_{A}$ denotes the indicator function of the set $A$. Therefore, the integration over $\theta$ in Eq. (12) should be performed over the interval $\left[\theta_{0}, \pi-\theta_{0}\right]$. This however, leads to the correction of the order of $(k f)^{-4}$ which is completely irrelevant from an experimental point of view. It is easy to notice that while calculating the integral in Eq. (14) in the intervals $\left[0, \theta_{0}\right]$ and $\left[\pi-\theta_{0}, \pi\right]$. In the experiment prepared by the Erlangen group, the focal length is of order of $f=2 \mathrm{~mm}$ and the wavelength of $\lambda=250 \mathrm{~nm}$, which amounts to $k f \simeq 10^{4}$ [10] and thus $\theta_{0}$ is small. Therefore, we can replace $\sin \theta$ by $\theta$. It is rather obvious that the same is true for any reasonable choice of the boundary conditions, because the smallness of this correction is entirely due to the large value of $k f$.

Hence, the only relevant modification of the spontaneous emission rate due to the presence of the mirror is the replacement of the plane traveling waves $f_{k}(\vec{n}, \vec{r})=e^{i k \vec{n} \cdot \vec{r}}$ by the standing waves

$$
f_{k}(\vec{n}, \vec{r})=\sqrt{2} \sin (k \vec{n} \cdot(\vec{r}-\vec{f})),
$$

where the factor $\sqrt{2}$ ensures the completeness condition. Eq. (25) implies that the electric field vanishes at the point $\mathrm{P}$ for any mode from Eq. (11). It leads to the final expression for the spontaneous emission rate in the presence of a conducting parabolic mirror

$$
\begin{aligned}
& \tilde{\Gamma}(k ; x, y, z)=\frac{1}{2 \pi^{2}} \frac{d^{2} k^{3}}{2 \hbar \epsilon_{0}} \int_{0}^{2 \pi} d \varphi \int_{0}^{\pi} d \theta \sin ^{3} \theta \\
& \sin ^{2}\{k[(x \cos \varphi+y \sin \varphi) \sin \theta+(z+f) \cos \theta]\} .
\end{aligned}
$$


If the atom is placed at a distance from the point $\mathrm{P}$ much larger than $\lambda$, the interference factor $\sin ^{2}(\ldots)$ is averaged to $1 / 2$ and the standard free space result (Eq. (15)) is recovered.

For the atom located at the mirror axis $x=y=0$, the integral in Eq. (26) simplifies to

$$
\tilde{\Gamma}(k ; z)=\eta \Gamma_{0}(k),
$$

where the correction to the free space decay rate is equal to

$$
\eta=\left(1+3 \frac{\cos (2 k(z+f))}{4 k^{2}(z+f)^{2}}-3 \frac{\sin (2 k(z+f))}{8 k^{3}(z+f)^{3}}\right) .
$$

The correction $\eta$ is evaluated for $f=2 \mathrm{~mm}$ and depicted in Fig. 2. Its value at the focal point becomes significant for small values of the wavevector $k$ (thus large values of the wavelength $\lambda$ ), corresponding to the condition $|z+f|<\lambda$, i.e. for the atom which is close the the mirror surface (within the distance of $\lambda)$. However, if $k$ gets large, then the $\eta$ fluctuations are shifted towards the mirror surface and take place only at a length of the order of the wavelength. Far away from the mirror all the fluctuations vanish, $\eta=1$, and thus we observe a free-space decay rate, also at the focus. According to the figures, in the planned experiment [10, 20] the changes in the decay rate could be observable on a scale of $100 \mathrm{~nm}$, but only within the distance of the wavelength from the mirror surface.

\section{Conclusions}

We rigorously analyzed the modification of the electromagnetic vacuum structure around an atom trapped at the focus of a parabolic metallic mirror. We assumed that the atomic dipole moment is parallel to the mirror axis. For a focal length which is large, compared to the wavelength of the photon emitted during the atomic transition, the total spontaneous emission rate does not differ from its free-space value essentially. However, the presence of the parabolic boundary conditions may be revealed in a different way. Since the energy distribution among different modes is sensitive to the precise position of the atom, one should observe interference effects on the screen perpendicular to the mirror axis away from the focal point. They are analogous to those observed in the experiment with an atom trapped in front of a flat mirror [15. The dipole radiation has to obey the boundary condition: the field has to vanish on the mirror surface, only those modes will contribute to the pattern on the screen which fulfill this condition. The other modes will be suppressed and will give rise to dark fringes. The detailed structure of the fringes depends on the value $k f$ and the precise position of the atom. 

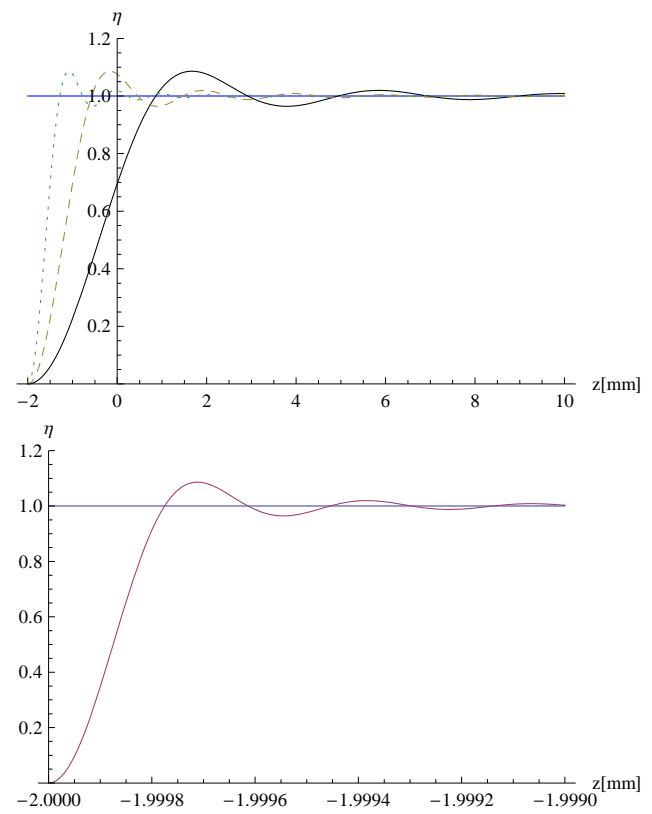

Fig. 2: The spontaneous emission rate corrections evaluated for small values of the wavevector (the top figure): $k=0.25 \pi \mathrm{mm}^{-1}$ - the solid line, $k=0.5 \pi$ $\mathrm{mm}^{-1}$ - the dashed line, $k=\pi \mathrm{mm}^{-1}$ - the dotted line and for large value of $k=10^{4} \mathrm{~mm}^{-1}$ (the bottom figure). 


\section{Acknowledgments}

This work was supported by the EU 7FP Marie Curie Career Integration Grant No. 322150 "QCAT", MNiSW grant No. 2012/04/M/ST2/00789 and

FNP Homing Plus project. R.A. is supported by the Polish research network LFPPI. The authors thank G. Leuchs, L. L. Sánchez-Soto and G. Alber for stimulating discussions.

\section{Bibliography}

1. J. I. Cirac, P. Zoller, H. J. Kimble, and H. Mabuchi, Phys. Rev. Lett. 78 (1997), 3221.

2. L.-M. Duan, M. D. Lukin, J. I. Cirac, and P. Zoller, Nature 414 (2001), 413.

3. X. Maitre et al, Phys. Rev. Lett. 79 (1997), 769.

4. C. J. Hood, T. W. Lynn, A. C. Doherty, A. S. Parkins, and H. J. Kimble, Science 287 (2000), 1447.

5. P. W. H. Pinkse, T. Fischer, P. Maunz, and G. Rempe, Nature 404 (2000), 365.

6. N. Lindlein, R. Maiwald, H. Konermann, M. Sondermann, U. Peshel, G. Leuchs, Laser Phys. 17 (2007), 927.

7. I. Gerhardt, G. Wrigge, P. Bushev, G. Zumofen, M. Agio, R. Pfab, and V. Sandoghdar, Phys. Rev. Lett. 98 (2007), 033601.

8. G. Zumofen, N. M. Mojarad, V. Sandoghdar, and M. Agio, Phys. Rev. Lett. 101 (2008), 180404.

9. M. K. Tey, Z. Chen, S. A. Aljunid, B. Chng, F. Huber, G. Maslennikov, and Ch. Kurtsiefer, Nature Phys. 4 (2008), 924.

10. M. Stobińska, G. Alber, and G. Leuchs, EPL 86 (2009), 14007.

11. M. Sondermann, R. Maiwald, H. Konermann, N. Lindlein, U. Peschel and G. Leuchs, App. Phys. B, 89 (2007), 489.

12. G. Alber, Phys. Rev. A 46 (1992), R5338.

13. K. H. Drexhage, Sci. Am. 222 (1970), 108.

14. U. Dorner and P. Zoller, Phys. Rev. A 66 (2002), 023816.

15. J. Eschner, Ch. Raab, and R. Blatt, Nature 413 (2001), 495.

16. R. Alicki, OSID 16 (2009), 49.

17. H. Morawitz, Phys. Rev. 187 (1969), 1792.

18. C. P. Boyer, E. G. Kalnins, and W. Miller, Nagoya Math. J. 60 (1976), 35.

19. J. U. Nockel, G. Bourdon, E. Le Ru, R. Adams, I. Robert, J.-M. Moison, and I. Abram, Phys. Rev. E 62 (2000), 8677.

20. M. Stobińska, M. Sondermann, G. Leuchs, Opt. Commun., 283 (2010), 737. 